Draft version August 25, 2018

Preprint typeset using $\mathrm{LAT}_{\mathrm{E}} \mathrm{X}$ style emulateapj v. 11/12/01

\title{
DARK ENERGY CONSTRAINTS FROM BARYON ACOUSTIC OSCILLATIONS
}

\author{
YUN WANG ${ }^{1}$ \\ Draft version August 25, 2018
}

\begin{abstract}
Baryon acoustic oscillations (BAO) in the galaxy power spectrum allows us to extract the scale of the comoving sound horizon at recombination, a cosmological standard ruler accurately determined by the cosmic microwave background anisotropy data. We examine various issues important in the use of BAO to probe dark energy. We find that assuming a flat universe, and priors on $\Omega_{m}, \Omega_{m} h^{2}$, and $\Omega_{b} h^{2}$ as expected from the Planck mission, the constraints on dark energy parameters $\left(w_{0}, w^{\prime}\right)$ scale much less steeply with survey area than (area $)^{-1 / 2}$ for a given redshift range. The constraints on the dark energy density $\rho_{X}(z)$, however, do scale roughly with $(\text { area })^{-1 / 2}$ due to the strong correlation between $H(z)$ and $\Omega_{m}$ (which reduces the effect of priors on $\Omega_{m}$ ). Dark energy constraints from BAO are very sensitive to the assumed linear scale of matter clustering and the redshift accuracy of the survey. For a BAO survey with $0.5 \leq z \leq 2, \sigma(R)=0.4$ (corresponding to $k_{\max }(z=0)=0.086 h \mathrm{Mpc}^{-1}$ ), and $\sigma_{z} /(1+z)=0.001,\left(\sigma_{w_{0}}, \sigma_{w^{\prime}}\right)=(0.115,0.183)$ and $(0.069,0.104)$ for survey areas of $1000(\mathrm{deg})^{2}$ and $10000(\mathrm{deg})^{2}$ respectively. We find that it is critical to minimize the bias in the scale estimates in order to derive reliable dark energy constraints. For a 1000 (10000) square degree BAO survey, a $1 \sigma$ bias in $\ln H(z)$ leads to a $2 \sigma(3 \sigma)$ bias in $w^{\prime}$. The bias in $w^{\prime}$ due to the same scale bias from $\ln D_{A}(z)$ is slightly smaller and opposite in sign. The results from this paper will be useful in assessing different proposed $\mathrm{BAO}$ surveys and guiding the design of optimal dark energy detection strategies.
\end{abstract}

Subject headings: Cosmology

\section{INTRODUCTION}

The most intriguing mystery in cosmology today is the nature of the dark energy that is driving the accelerated expansion of the universe as evidenced by supernova (Riess et al. 1998; Perlmutter et al. 1999) and other data. Although current observational data are consistent with a cosmological constant (see for example, Wang \& Tegmark (2004, 2005); Daly \& Djorgovski (2005); Jassal, Bagla, \& Padmanabhan (2005)), viable dark energy models abound. See for example, Freese et al. (1987); Linde (1987); Peebles \& Ratra (1988); Wetterich (1988); Frieman et al. (1995); Caldwell, Dave \& Steinhardt (1998); Sahni \& Habib (1998); Parker \& Raval (1999); Dvali, Gabadadze, \& Porrati (2000); Mersini, Bastero-Gil, \& Kanti (2001); Freese \& Lewis (2002); Carroll et al. (2004); Onemli \& Woodard (2004); Alam \& Sahni (2005); Cai, Gong, \& Wang (2005); Cardone et al. (2005); Kolb, Matarrese, \& Riotto (2005); Martineau \& Brandenberger (2005). Padmanabhan (2003) and Peebles \& Ratra (2003) contain reviews of many models.

The powerful complementarity of the cosmic microwave anisotropy (CMB) and galaxy survey data in precision cosmology has long been noted (see for example, Bahcall et al. (1999), Eisenstein, Hu, \& Tegmark (1999), and Wang, Spergel, \& Strauss (1999)). An important development in this complementarity is to use the baryonic acoustic oscillations (BAO) in the galaxy power spectrum as a cosmological standard ruler to probe dark energy (Blake \& Glazebrook 2003; Seo \& Eisenstein 2003), made possible by accurate determination of the standard ruler scale by CMB data from WMAP (Bennett et al. 2003; Spergel et al. 2003) and Planck ${ }^{2}$.

The "wavelength" of the baryonic acoustic oscillations in $k$-space is determined by $k_{A}=2 \pi / s$, where $s$ is the comoving sound horizon at the drag epoch (when baryons are released from the Compton drag of the photons ${ }^{3}$ ),

$$
s=\int_{0}^{t_{d}} c_{s} d t / a=H_{0}^{-1} \int_{z_{d}}^{\infty} d z \frac{c_{s}}{H(z)}
$$

The sound speed is

$$
\begin{aligned}
c_{s} & =\frac{1}{\sqrt{3\left(1+R^{b}\right)}}, \\
R^{b} & =\frac{3 \rho_{b}}{4 \rho_{\gamma}}=31.5 \Omega_{b} h^{2}\left(T_{C M B} / 2.7 \mathrm{~K}\right)^{-4}(z / 1000)^{-1},
\end{aligned}
$$

and the Hubble parameter is

$$
H(z)=\sqrt{\Omega_{m}(1+z)^{3}+\Omega_{\text {rad }}(1+z)^{4}+\Omega_{k}(1+z)^{2}+\Omega_{X} X(z)},
$$

with $X(z) \equiv \rho_{X}(z) / \rho_{X}(0)$ denoting the dark energy density function. For a cosmological constant, $X(z)=1$ and $\Omega_{X}=\Omega_{\Lambda}$. Since $z_{d} \gg 1$, we have

$$
\begin{aligned}
s & \simeq \frac{H_{0}^{-1}}{\sqrt{\Omega_{m}}} \int_{0}^{a_{d}} d a \frac{c_{s}}{\sqrt{a+a_{e q}}} \\
& =\frac{1}{\sqrt{\Omega_{m} H_{0}^{2}}} \frac{2 c}{\sqrt{3 z_{e q} R_{e q}^{b}}} \ln \frac{\sqrt{1+R_{d}^{b}}+\sqrt{R_{d}^{b}+R_{e q}^{b}}}{1+\sqrt{R_{e q}^{b}}},(4)
\end{aligned}
$$

where "d" and "eq" refer to the drag epoch and matterradiation equality respectively, with $z_{d} \sim 1089$, and $z_{e q}=$ $2.5 \times 10^{4} \Omega_{m} h^{2}\left(T_{C M B} / 2.7 \mathrm{~K}\right)^{-4}$. Hence the "wavelength" of the baryonic oscillations depends very strongly on $\Omega_{m}$,

${ }^{1}$ Department of Physics \& Astronomy, Univ. of Oklahoma, 440 W Brooks St., Norman, OK 73019; email: wang@nhn.ou.edu (Jan 8, 2006)

2 See Planck mission blue book at http://www.rssd.esa.int/SA/PLANCK/docs/Bluebook-ESA-SCI(2005)1.pdf

${ }^{3}$ For $\Omega_{b} h^{2} \lesssim 0.03$, the drag epoch follows the last scattering of the photons (Eisenstein \& Hu 1998). 
but has negligible dependence on dark energy (which only became important recently, at redshifts a few and less). It can therefore be used as a standard ruler to probe dark energy.

The systematic effects of BAO as a standard ruler are: bias between luminous matter and matter distributions, nonlinear effects, and redshift distortions (Blake \& Glazebrook 2003; Seo \& Eisenstein 2003). Cosmological N-body simulations are required to quantify these effects (Angulo et al. 2005; Seo \& Eisenstein 2005; Springel et al. 2005; White 2005). Fisher matrix formalism is useful in investigating the impact of various effects and assumptions that can be parametrized in the observed galaxy power spectrum (Seo \& Eisenstein 2003).

In this paper, we examine various issues important in the use of baryon acoustic oscillations (BAO) to probe dark energy using the Fisher matrix formalism. In particular, we extend the work of Seo \& Eisenstein (2003) by deriving the biases in dark energy parameters $\left(w_{0}, w^{\prime}\right)$ due to systematic errors in the estimated scales. Throughout this paper, we assume spatial flatness as motivated by inflation and consistent with current CMB data.

Sec. 2 describes the method used in our calculations. We present results in Sec.3 and summarize in Sec.4.

\section{THE METHOD}

\subsection{Error estimation using Fisher matrix}

In estimating the expected errors in dark energy parameters, we follow Seo \& Eisenstein (2003) in using the Fisher matrix formalism (see discussion in Sec.4). We provide details from our calculations for the convenience of readers who wish to reproduce our results.

The comoving sizes of an object or feature at redshift $z$ in line-of-sight $\left(r_{\|}\right)$and transverse $\left(r_{\perp}\right)$ directions are related to the observed sizes $\Delta z$ and $\Delta \theta$ by the Hubble parameter $H(z)$ and angular diameter distance $D_{A}(z)$ :

$$
r_{\|}=\frac{c \Delta z}{H(z)}, \quad r_{\perp}=(1+z) D_{A}(z) \Delta \theta,
$$

The true scale of the baryonic acoustic oscillations (the comoving sound horizon at recombination) is known. Hence if we can measure the "wavelength" of the baryonic acoustic oscillations in the radial direction in successive redshift slices, we obtain estimates of the cosmic expansion history as a free function of $z$. While the measurement of the "wavelength" of the baryonic acoustic oscillations in the transverse direction gives us an estimate of the angular diameter distance $D_{A}(z)$ as a free function of $z$.

The accuracy of the power spectrum measurement (Feldman, Kaiser, \& Peacock 1994; Tegmark 1997) is

$$
\frac{\sigma_{P}}{P}=2 \pi \sqrt{\frac{2}{V_{\text {survey }} k^{2} \Delta k \Delta \mu}}\left(1+\frac{1}{n P}\right),
$$

where $P$ is the average band power, $V_{\text {survey }}$ is the total survey volume, $\Delta k$ is the range of wavenumber $k$ averaged over, $\Delta \mu$ is the range of the cosine of the angle between the wavevector $\mathbf{k}$ and the line of sight, and $n$ is the comoving number density of observed galaxies. It is reasonable to assume $n P \sim 3$ (Blake \& Glazebrook 2003; Seo \& Eisenstein 2003). This gives an estimate of how accurately the "wavelength" of the baryonic oscillations can be recovered. Note that in our calculation, Eq.(6) is only used to get an estimate of how accurately the power spectrum is determined, and not used to derive constraints on dark energy and cosmological parameters.

Assuming that the likelihood function for the band powers of a galaxy redshift survey is Gaussian, the Fisher matrix can be approximated as (Tegmark 1997)

$$
F_{i j}=\int_{k_{\min }}^{k_{\max }} \frac{\partial \ln P(\mathbf{k})}{\partial p_{i}} \frac{\partial \ln P(\mathbf{k})}{\partial p_{j}} V_{e f f}(\mathbf{k}) \frac{d \mathbf{k}^{3}}{2(2 \pi)^{3}}
$$

where the derivatives are evaluated at parameter values of the fiducial model and $V_{e f f}$ is the effective volume of the survey

$$
\begin{aligned}
V_{\text {eff }}(k, \mu) & =\int\left[\frac{n(\mathbf{r}) P(k, \mu)}{n(\mathbf{r}) P(k, \mu)+1}\right]^{2} d \mathbf{r}^{3} \\
& =\left[\frac{n P(k, \mu)}{n P(k, \mu)+1}\right]^{2} V_{\text {survey }},
\end{aligned}
$$

where the comoving number density $n$ has been taken to be constant in position. Here $\mu=\mathbf{k} \cdot \hat{\mathbf{r}} / k$, with $\hat{\mathbf{r}}$ denoting the unit vector along the line of sight; $\mathbf{k}$ is the wavevector with $|\mathbf{k}|=k$. Following Blake \& Glazebrook (2003) and Seo \& Eisenstein (2003), we take $k_{\min }=0$, and $k_{\max }$ given by requiring that $\sigma(R) \lesssim 0.5$ for $R=\pi /\left(2 k_{\max }\right)$ (to ensure that we are only considering the linear regime).

The observed power spectrum is reconstructed using a particular reference cosmology, including the effects of bias and redshift distortions (Seo \& Eisenstein 2003):

$$
\begin{aligned}
& P_{o b s}\left(k_{r e f \perp}, k_{r e f \|}\right)=\frac{\left[D_{A}(z)_{r e f}\right]^{2} H(z)}{\left[D_{A}(z)\right]^{2} H(z)_{r e f}} b^{2}\left(1+\beta \frac{k_{\|}^{2}}{k_{\perp}^{2}+k_{\|}^{2}}\right)^{2} . \\
& \cdot\left[\frac{G(z)}{G(z=0)}\right]^{2} P_{\text {matter }}(k \mid z=0)+P_{\text {shot }}(9)
\end{aligned}
$$

The values in the reference cosmology are denoted by the subscript "ref", while those in the true cosmology have no subscript. Note that

$$
k_{r e f \perp}=k_{\perp} D_{A}(z) / D_{A}(z)_{r e f}, \quad k_{r e f \|}=k_{\|} H(z)_{r e f} / H(z) .
$$

The linear redshift distortion $\beta$ is computed from the bias $b$ for fiducial values of the observed galaxy clustering, $\beta=\Omega_{m}^{0.6} / b . G(z)$ is the linear growth factor. We normalize the power spectrum to $\mathrm{CMB}$ data from $\mathrm{COBE}$ (Eisenstein \& Hu 1998).

Care needs to be taken in using Eq.(9) to compute the derivatives of $P(\mathbf{k})$ needed for the Fisher matrix in Eq.(7). Note that $P_{\text {matter }}(k \mid z=0)$ depends on $H(z)$ and $D_{A}(z)$ through $k$ (see Eq.(10)).

For a redshift slice with mean redshift $z$, the estimated parameters (assumed to be constant in the redshift slice) are the Hubble parameter $H(z)$, angular diameter distance $D_{A}(z)$, linear redshift distortion $\beta$, linear growth function $G(z)$, and an unknown shot noise $P_{\text {shot }} \cdot{ }^{4}$ These are estimated simultaneously with $\Omega_{m}, \Omega_{m} h^{2}$, and $\Omega_{b} h^{2}$. The total number of parameters is $5 N+3$ for a BAO survey divided into $N$ redshift slices.

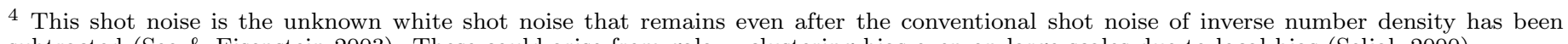
subtracted (Seo \& Eisenstein 2003). These could arise from galaxy clustering bias even on large scales due to local bias (Seljak 2000). 
Note that the same cutoff scales $k_{\max }(z)$ need to be used for computing all the Fisher matrix elements, including those of $\Omega_{m}, \Omega_{m} h^{2}$, and $\Omega_{b} h^{2}$. To implement this, calculate $F_{i j}$ in each redshift slice using the appropriate $k_{\max }(z)$, then sum over all the redshift slices for $\Omega_{m}, \Omega_{m} h^{2}$, and $\Omega_{b} h^{2}$. If a fixed cutoff scale is used for computing $F_{i j}$ for the cosmological parameters, the degeneracy between $\left(H(z), D_{A}(z)\right)$ from the redshift slices and the cosmological parameters (especially $\Omega_{m}$ ) will be artificially broken, leading to significant under-estimates of errors in $\left(H(z), D_{A}(z)\right)$ and the dark energy parameters.

To obtain constraints on $\left(w_{0}, w^{\prime}, \Omega_{m}, \Omega_{m} h^{2}\right)$, the parameters of interest are $\mathbf{p}=\left(\ln H^{i}, \ln D_{A}^{i}, i=1,2, \ldots, N\right.$; $\left.\Omega_{m}, \Omega_{m} h^{2}\right)$. First marginalize over $\left(\beta, G(z), P_{\text {shot }}\right)$ in each redshift slice as well as $\Omega_{b} h^{2}$ by taking the submatrix (of the parameters of interest) of the inverse of the full Fisher matrix, then invert it to obtain the Fisher matrix of the parameters of interest, $F^{s u b}$. The Fisher matrix of $\mathbf{q}=\left(w_{0}, w^{\prime}, \Omega_{m}, \Omega_{m} h^{2}\right)$ is obtained by equating the $\log$ likelihood functions $(\ln \mathcal{L}(\mathbf{p})=\ln \mathcal{L}(\mathbf{q}))$, then taking derivatives with respect to $\mathbf{q}$ on both sides. This gives

$$
F_{D E, i j}=\sum_{\alpha, \beta} \frac{\partial p_{\alpha}}{\partial q_{i}}\left(F^{s u b}\right)_{\alpha \beta} \frac{\partial p_{\beta}}{\partial q_{j}}
$$

\subsection{Bias in dark energy parameters}

We now extend the work of Seo \& Eisenstein (2003) by deriving the bias in dark energy parameters due to systematic errors that bias the extracted standard ruler scale.

The standard ruler scale is measured using $H(z)$ and $D_{A}(z)$ in each redshift slice. First, we marginalize over all other parameters by taking the $\left(\ln H^{i}, \ln D_{A}^{i}, i=1,2\right.$, ..., $N$ ) submatrix of the inverse of the full Fisher matrix, then invert it to obtain the Fisher matrix relevant for scale determination, $F^{\text {scale }}$.

To compute the bias in $\left(w_{0}, w^{\prime}\right)$ due to biases in $\left(\ln H^{i}\right.$, $\left.\ln D_{A}^{i}, i=1,2, \ldots, N\right)$, find the $\left(w_{0}, w^{\prime}\right)$ Fisher matrix by contracting $F^{\text {scale }}\left(\ln H^{i}, \ln D_{A}^{i}, i=1,2, \ldots, N\right)$ to $F_{D E, i j}^{\text {scale }}$ $\left(w_{0}, w^{\prime}\right)$ (see derivation of Eq.(11)):

$$
F_{D E, i j}^{s c a l e}=\sum_{\alpha, \beta} \frac{\partial p_{\alpha}}{\partial q_{i}}\left(F^{\text {scale }}\right)_{\alpha \beta} \frac{\partial p_{\beta}}{\partial q_{j}}
$$

To relate the biases in $\left(w_{0}, w^{\prime}\right)$ to biases in $\left(\ln H^{i}\right.$, $\left.\ln D_{A}^{i}, i=1,2, \ldots, N\right)$, equate the log likelihood functions $\ln \mathcal{L}^{\text {scale }}\left(\mathbf{p}-\mathbf{p}_{m}^{\prime}\right)=\ln \mathcal{L}^{\text {scale }}\left(\mathbf{q}-\mathbf{q}_{m}^{\prime}\right)+$ const., subtract $\ln \mathcal{L}^{\text {scale }}\left(\mathbf{p}-\mathbf{p}_{m}\right)=\ln \mathcal{L}^{\text {scale }}\left(\mathbf{q}-\mathbf{q}_{m}\right)$ from it, where $\mathbf{p}_{m}^{\prime}=\mathbf{p}_{m}+\delta \mathbf{p}_{m}$, and $\mathbf{q}_{m}^{\prime}=\mathbf{q}_{m}+\delta \mathbf{q}_{m}$ are the biased mean values. This gives

$$
\begin{aligned}
\left(\mathbf{q}-\mathbf{q}_{m}\right)_{i}^{T} F_{D E, i j}^{\text {scale }}\left(\delta \mathbf{q}_{m}\right)_{j}= & \left(\mathbf{p}-\mathbf{p}_{m}\right)_{\alpha}^{T}\left(F^{\text {scale }}\right)_{\alpha \beta}\left(\delta \mathbf{p}_{m}\right)_{\beta} \\
& + \text { const. }
\end{aligned}
$$

Taking derivative with respect to $q_{i}$ on both sides gives

$$
F_{D E, i j}^{\text {scale }}\left(\delta \mathbf{q}_{m}\right)_{j}=\frac{\partial p_{\alpha}}{\partial q_{i}}\left(F^{\text {scale }}\right)_{\alpha \beta}\left(\delta \mathbf{p}_{m}\right)_{\beta} .
$$

Hence

$$
\left(\delta \mathbf{q}_{m}\right)_{i}=\left(F_{D E}^{\text {scale }}\right)_{i j}^{-1} \frac{\partial p_{\alpha}}{\partial q_{j}}\left(F^{\text {scale }}\right)_{\alpha \beta}\left(\delta \mathbf{p}_{m}\right)_{\beta},
$$

where $\delta \mathbf{p}_{m}$ are the biases in $\left(\ln H^{i}, \ln D_{A}^{i}, i=1,2, \ldots, N\right)$, and summation is implied over repeated indices.
A known example of BAO systematic bias is a bias in the dilation parameter $\alpha=k_{\text {ref }} / k_{\text {true }}$ for spherically averaged galaxy power spectrum. Seo \& Eisenstein (2005) has shown that $\alpha$ is biased slightly above 1 in currently used methods for accounting the erasure of baryonic features due to nonlinear effects (see also White (2005)).

\section{RESULTS}

We consider a BAO survey in the redshift range of $0.5 \leq z \leq 2$, which can be carried out by obtaining the spectra of $\mathrm{H} \alpha$ emission line galaxies with spectrographs covering the wavelength range of $1-2 \mu \mathrm{m}$. Note that $0.5 \leq z \leq 2$ is the redshift range with the most sensitivity to the time variation of dark energy (Glazebrook \& Blake 2005), and easily accessible by a space mission with simple spectroscopic instrumentation. Examples include the BOP MIDEX concept (Glazebrook et al. 2005), and the JEDI mission concept for JDEM (Wang et al. 2004; Crotts et al. 2005).

Note that our results should qualitatively apply to other BAO surveys, in particular, ground based surveys. Ground based BAO surveys, for example, HETDEX (Hill et al. 2004) and WFMOS (Glazebrook et al. 2005), have been planned and will likely occur before a BAO survey from space. Both ground and space BAO surveys will be needed to establish BAO as a dark energy probe, and to obtain accurate and high precision dark energy constraints.

The fiducial cosmological model we have assumed is $n_{S}=1, h=0.65, \Omega_{m}=0.3, \Omega_{\Lambda}=0.7, \Omega_{b}=0.05$. We assume the following priors as expected from CMB data from the Planck mission: $\sigma_{\Omega_{m}}=0.01, \sigma_{\Omega_{m} h^{2}} /\left(\Omega_{m} h^{2}\right)=0.01$, and $\sigma_{\Omega_{b} h^{2}} /\left(\Omega_{b} h^{2}\right)=0.01$. These parameters are estimated simultaneously with $H(z), D_{A}(z), \beta, G(z)$, and $P_{\text {shot }}$ from seven redshift slices. Each redshift slice has the thickness $\Delta z=0.2$, except for the first redshift slice which has $\Delta z=0.3(0.5<z<0.8)$. We assume a cutoff scale $k_{\max }$ given by $k_{\max }=\pi /(2 R)$, with $\sigma(R)=0.4$ (except for Fig.2, where $\sigma(R)$ is varied between 0.3 and 0.5 ), and a survey redshift accuracy of $\sigma_{z} /(1+z)=0.001$ (except for Fig.3, where $\sigma_{z} /(1+z)$ is varied between 0 and 0.02$)$. We have conservatively taken the galaxy bias to be 1 (higher bias leads to smaller errors in estimated dark energy parameters ${ }^{5}$ ), and assumed the comoving number density of observed galaxies such that $n P=3$ following Blake \& Glazebrook (2003).

The dark energy equation of state is parametrized by $w(z)=w_{0}+w^{\prime} z$ for $z \leq 2$. It is better to constrain dark energy density $\rho_{X}(z)$ instead of dark energy equation of state (Wang \& Garnavich 2001; Tegmark 2002; Wang \& Freese 2004). This is because dark energy density is more closely related to observables and thus more tightly constrained. We use dark energy equation of state parameters in this paper for easy comparison with the work by others. Note that the dark energy observables here are $H(z)$, $D_{A}(z)$ from each redshift slice. The errors in $H(z), D_{A}(z)$ are propagated into the errors in $w_{0}$ and $w^{\prime}$ by contracting the covariance matrix (see Eq.(11)). We give constraints on $\rho_{X}(z)$ in Fig.5.

Fig.1 shows the $1 \sigma$ errors in $w_{0}$ and $w^{\prime}$ as function of survey area $(0.5 \leq z \leq 2)$. In the absence of the priors, the

\footnotetext{
5 This is a small effect when $n P=3$ and the cosmic variance error dominates.
} 
error bars on dark energy parameters scale as (area $)^{-1 / 2}$ for a given redshift range. Note that with Planck priors on cosmological parameters, the constraints on dark energy parameters scale much less steeply with survey area than $(\text { area })^{-1 / 2}$ for a given redshift range. For a BAO survey with $0.5 \leq z \leq 2,\left(\sigma_{w_{0}}, \sigma_{w^{\prime}}\right)=(0.115,0.183)$ and $(0.069,0.104)$ for survey areas of $1000(\mathrm{deg})^{2}$ and 10000 $(\operatorname{deg})^{2}$ respectively. The smaller the survey area, the greater the impact of the priors on cosmological parameters.

Dark energy constraints from BAO are very sensitive to the linear scale of matter clustering. Fig. 2 shows the $1 \sigma$ errors in $w_{0}$ and $w^{\prime}$ as functions of $\sigma(R)$ for survey areas of 1000 and $10000(\mathrm{deg})^{2}$. The lowest panel in Fig.2 gives the correspondence between $\sigma(R)$ and the cutoff wavenumber $k_{\max }$ at $z=0$. Note that it is important to compare results for the same $k_{\max }$ at $z=0$, since the same $k_{\max }(z=0)$ may correspond to different $\sigma(R)$ if different normalizations are used for the power spectrum.

Dark energy constraints from BAO are very sensitive to the redshift accuracy of the survey. Fig. 3 shows the $1 \sigma$ errors in $w_{0}$ and $w^{\prime}$ as functions of $\sigma_{z} /(1+z)$ for survey areas of 1000 and 10000 square degrees. The redshift uncertainty $\sigma_{z}$ leads to a radial smearing $\sigma_{r}=c \sigma_{z} / H(z)$; which modifies the matter power spectrum in Eq.(9) as follows (see for example Peacock (1999)):

$$
P_{o b s}\left(\mathbf{k} \mid \sigma_{z}\right)=P_{o b s}\left(\mathbf{k} \mid \sigma_{z}=0\right) \exp \left(-k_{\|}^{2} \sigma_{r}^{2}\right) .
$$

Fig.4 shows $\sigma_{H} / H$ and $\sigma_{D_{A}} / D_{A}$ in each of the redshift slices, for $\sigma_{z} /(1+z)=0.001,0.01$, and 0.02 , for 1000 and $10000(\mathrm{deg})^{2}$ surveys with $0.5 \leq z \leq 2$. These represent the accuracy with which model-independent constraints on dark energy can be placed. These are also relevant for estimating likely biases in $\left(w_{0}, w^{\prime}\right)$ due to biases in scale estimates.

Fig.5 shows the constraints on the dark energy density $X(z)=\rho_{X}(z) / \rho_{X}(0)$ corresponding to Fig.4 (with the same assumptions and the same line types). The errors on $X(z)$ have been propagated from the errors on $H(z)$ in each redshift slice and the error on $\Omega_{m}$ (see Eq.[3]), with the covariance between $H(z)$ and $\Omega_{m}$ included. Note that $X(z)$ does scale roughly as (area) $)^{-1 / 2}$, because the strong covariance between $\Omega_{m}$ and $H(z)$ makes the effects of the cosmological priors less important.

Table 1 gives biases in $\left(w_{0}, w^{\prime}\right)$ due to biases in $\left(\ln H^{i}\right.$, $\ln D_{A}^{i}$ ) for 1000 and 10000 square degree BAO surveys with $\sigma(R)=0.4$. Note that " $1 \sigma$ " denotes setting the bias in $\left(\ln H^{i}, \ln D_{A}^{i}\right)$ to $1 \sigma$ errors in $\left(\ln H^{i}, \ln D_{A}^{i}\right)$. The sign of the biases in $\left(\ln H^{i}, \ln D_{A}^{i}\right)$ in Table 1 are chosen to correspond to scale biases in the same direction, the direction of wavenumber dilation $\alpha=k_{r e f} / k_{\text {true }}>1$ as found by Seo \& Eisenstein (2005).

If the dilation is assumed to be uniform in all directions, then the bias in $\ln H(z)$ and $\ln D_{A}(z)$ will be equal and opposite in sign, leading to minimized biases in $w_{0}$ and $w^{\prime}$. However, the bias in the estimated $\ln H(z)$ should depend on the modeling of redshift distortions and on the redshift accuracy of the survey. Hence it is likely that dilation in wavenumber will not be uniform in all directions. For this reason, we give the biases due to $\ln H(z)$ and $\ln D_{A}(z)$ sep- arately in Table 1. Cosmological N-body simulations are required to quantify the expected biases in $\ln H(z)$ and $\ln D_{A}(z)$.

\section{DISCUSSION AND SUMMARY}

Baryon acoustic oscillations (BAO) provide an important new method for probing dark energy. To extract reliable dark energy constraints from BAO surveys, we need to understand how the expected errors depend quantitatively on the assumptions made.

For simplicity and transparency, we use the Fisher information matrix formalism in this paper. Our results are consistent with those of Seo \& Eisenstein (2003), and agree to about $10 \%$ with those found using Monte Carlo methods by Blake \& Glazebrook (2003) and Glazebrook \& Blake (2005), for the same $k_{\max }(z=0)$ and priors on cosmological parameters. Although Fisher matrix yields the smallest possible error bars, we have made the most conservative assumptions. In deriving the dark energy constraints, we marginalize over many additional physical parameters (Seo $\&$ Eisenstein 2003): the linear redshift distortion $\beta$, linear growth function $G(z)$, and an unknown shot noise $P_{\text {shot }}$ in each redshift slice; the matter density ratio $\Omega_{m}$, the matter density $\Omega_{m} h^{2}$, and the baryon density $\Omega_{b} h^{2}$. This approach seems to work rather well, as Seo \& Eisenstein (2005) found that their results from cosmological N-body simulations are close to what they found using the Fisher matrix formalism (Seo \& Eisenstein 2003).

We have examined various issues important in the use of BAO to probe dark energy. In particular, we extend the work of Seo \& Eisenstein (2003) by deriving the biases in dark energy parameters $\left(w_{0}, w^{\prime}\right)$ due to systematic errors in the estimated scales.

We find that assuming priors on $\Omega_{m}, \Omega_{m} h^{2}$, and $\Omega_{b} h^{2}$ as expected from the Planck mission, the constraints on dark energy parameters $\left(w_{0}, w^{\prime}\right)$ scale much less steeply with survey area (for a given redshift range) than (area) $)^{-1 / 2}$ (which holds in the absence of the priors). For a BAO survey with $0.5 \leq z \leq 2, \sigma(R)=0.4$ (corresponding to $\left.k_{\text {max }}(z=0)=0.086 h \mathrm{Mpc}^{-1}\right)$, and $\sigma_{z} /(1+z)=0.001$, $\left(\sigma_{w_{0}}, \sigma_{w^{\prime}}\right)=(0.115,0.183)$ and $(0.069,0.104)$ for survey areas of $1000(\mathrm{deg})^{2}$ and $10000(\mathrm{deg})^{2}$ respectively (see Fig.1). This provides a useful guide in designing optimal survey strategies that provide robust and accurate dark energy constraints.

The constraints on the dark energy density $\rho_{X}(z)$, however, do scale roughly with (area) $)^{-1 / 2}$ due to the strong correlation between $H(z)$ and $\Omega_{m}$ (which reduces the effect of priors on $\left.\Omega_{m}\right)$. This is interesting since $\rho_{X}(z)$ provides direct and model-independent constraints on dark energy (Wang \& Garnavich 2001; Tegmark 2002; Wang \& Freese 2004).

The BAO constraints on the dark energy equation of state parameters $\left(w_{0}, w^{\prime}\right)$ are very sensitive to the assumed linear scale of matter clustering $k_{\max }(z)^{6}$ and the redshift accuracy of the survey $\sigma_{z} /(1+z)$ (see Figs.2-3). This is important to note since different proposed BAO surveys are often compared by their expected errors in $\left(w_{0}, w^{\prime}\right)$. Such comparisons are not appropriate unless the same assumptions are made about $k_{\max }(z)$, and the claimed

${ }^{6}$ Once realistic N-body simulations are available for calibrating the analysis of real data, we will be able to extract additional information on dark energy parameters by using the data from the quasi-linear regime. 
$\sigma_{z} /(1+z)$ can be demonstrated to be feasible. The latter is a key issue for surveys using photometric redshifts (Blake \& Glazebrook 2003; Seo \& Eisenstein 2003; Zhan \& Knox 2005).

It should be noted that the radial BAO are not observable for photometric redshift accuracies. The $H(z)$ measurement from using photometric redshifts is derived from the degree of damping of broad-band power in the radial direction on very large scales $\left(k<0.05 \mathrm{~h} \mathrm{Mpc}^{-1}\right)$. Hence this measurement is much less robust (more susceptible to systematic error) than the spectroscopic case, where the radial $\mathrm{BAO}$ are detectable and give $H(z)$ measurements directly.

We find that it is critical to minimize the bias in the scale estimates $\left(\ln H(z)\right.$ and $\left.\ln D_{A}(z)\right)$ in order to derive reliable dark energy constraints. For a 1000 (10000) sq deg BAO survey, a $1 \sigma$ bias in $\ln H(z)$ leads to a $2 \sigma(3 \sigma)$ bias in $w^{\prime}$. The bias in $w^{\prime}$ due to the same scale bias from $\ln D_{A}(z)$ is slightly smaller and opposite in sign. In addition to the scale bias due to the inaccurate modeling of nonlinear effects (which impacts the results in the linear regime, see Seo \& Eisenstein (2005)), systematic biases in the $H(z)$ measurement could arise when photo- metric redshifts are used. It is important to quantify the expected bias in $\ln H(z)$ and $\ln D_{A}(z)$ using cosmological N-body simulations. Note that $H(z)$ and $D_{A}(z)$ are measured as independent parameters. Since $D_{A}(z)$ is related to $H(z)$ through an integral for a given cosmological model in the absence of systematic biases, comparing the directly measured $D_{A}(z)$ to the $D_{A}(z)$ derived from the $H(z)$ measurement provides us with a cross-check to help model unknown systematic effects.

The planned BAO surveys from both ground and space telescopes will play an important role in unraveling the nature of dark energy. The results from in this paper will be useful in assessing different proposed BAO surveys and guiding the design of optimal dark energy detection strategies.

Acknowledgements I am grateful to Chris Blake for making his Monte Carlo results available to me for comparisons, and for useful discussions. I thank Max Tegmark, Dan Eisenstein, and Hee-Jong Seo for helpful discussions. This work was supported in part by NSF CAREER grants AST-0094335.

\section{REFERENCES}

Alam, U., \& Sahni, V. 2005, astro-ph/0511473

Angulo, R., et al. 2005, Mon.Not.Roy.Astron.Soc.Lett., 362, L25-L29

Bahcall,N., Ostriker, J.P., Perlmutter, S., \& Steinhardt, P.J. 1999, Science, 284, 1481

Blake, C.; and Glazebrook, K. 2003, ApJ, 594, 665

Bennett, C. L., et al. 2003, ApJS, 148, 1

Caldwell, R., Dave, R., \& Steinhardt, P.J., 1998, PRL, 80, 1582

Cardone, V.F., Tortora, C., Troisi, A., \& Capozziello, S. 2005, astro$\mathrm{ph} / 0511528$, Phys.Rev.D, in press

Carroll, S M, de Felice, A, Duvvuri, V, Easson, D A, Trodden, M \& Turner, M S, astro-ph/0410031

Cai, R., Gong, Y., \& Wang, B. 2005, hep-th/0511301

Crotts, A. et al. 2005, astro-ph/0507043, JEDI white paper submitted to the Dark Energy Task Force.

Daly,R. A.,\& Djorgovski, S. G. 2005, astro-ph/0512576.

Dvali, G., Gabadadze, G., \& Porrati, M. 2000, Phys.Lett. B485, 208

Eisenstein, D. \& Hu, W. 1998, ApJ, 496, 605

Eisenstein, D., Hu, W., \& Tegmark, M. 1999, ApJ, 518, 2

Feldman, H.A.; Kaiser, N.; \& Peacock, J.A. 1994, ApJ, 426, 23

Freese, K., et al., 1987, Nucl. Phys., B287, 797

Freese, K., Lewis, M. 2002, Phys.Lett. B540, 1

Frieman, J.A., Hill, C.T., Stebbins, A., and Waga, I., 1995, PRL, 75, 2077

Glazebrook, K. et al. 2005, astro-ph/0410037, New Astron.Rev. 49, 374

Glazebrook, K. \& Blake, C. 2005, ApJ, 631, 1

Glazebrook, K., et al. 2005, astro-ph/0507457, WFMOS white paper submitted to the Dark Energy Task Force.

Hill, G.J., et al. 2004, in proceedings of the Mitchell symposium.

Jassal, H.K., Bagla, J.S., Padmanabhan, T. 2005 , Mon.Not.Roy.Astron.Soc.Letters, 356, L11

Kolb, E.W., Matarrese, S., \& Riotto, A. 2005, astro-ph/0506534
Linde A D, "Inflation And Quantum Cosmology" in Three hundred years of gravitation, (Eds.: Hawking, S.W. and Israel, W., Cambridge Univ. Press, 1987), 604-630.

Martineau, P., \& Brandenberger, R., astro-ph/0510523

Mersini, L., Bastero-Gil, M., \& Kanti, P. 2001 Phys.Rev. D64, 043508

Onemli, V. K., \& Woodard, R. P. 2004, Phys.Rev. D70, 107301

Padmanabhan, T., 2003, Phys. Rep., 380, 235

Parker, L., and Raval, A., 1999, PRD, 60, 063512

Peacock, J.A., 1999, "Cosmological Physics", Cambridge University Press

Peebles, P.J.E., and Ratra, B., 1988, ApJ, 325, L17

Peebles, P.J.E., \& Ratra, B., 2003, Rev. Mod. Phys., 75, 559

Perlmutter, S. et al., 1999, ApJ, 517, 565

Riess, A. G, et al., 1998, Astron. J., 116, 1009

Sahni,V., \& Habib, S. 1998, Phys.Rev.Lett. 81, 1766

Seljak, U. 2000, MNRAS, 318, 203

Seo, H., Eisenstein, D.J. 2003, ApJ, 598, 720

Seo, H., Eisenstein, D.J. 2003, ApJ, 633, 575

Spergel, D.N., et al. 2003, ApJS, 148, 175

Springel, V., et al. 2005, Nature, 435, 629

Tegmark, M. 1997, PRL, 79, 3806

Tegmark, M. 2002, Phys. Rev. D66, 103507

Wang, Y., and Garnavich, P. 2001, ApJ, 552, 445

Wang, Y., et al. 2004 (the JEDI Team), BAAS, 36, 5, AAS205, 1328. See also http://jedi.nhn.ou.edu/.

Wang, Y., and Freese, K., astro-ph/0402208

Wang, Y., Spergel, D.N., \& Strauss, M. 1999, ApJ, 510, 20

Wang, Y., \& Tegmark, M. 2004, Phys. Rev. Lett., 92, 241302

Wang, Y., \& Tegmark, M. 2005, Phys. Rev. D 71, 103513

Wetterich, C., 1988, Nucl.Phys., B302, 668

White, M. 2005, Astropart.Phys. 24, 334

Zhan, H., \& Knox, L., astro-ph/0509260 
TABle 1

Biases in $\left(w_{0}, w^{\prime}\right)$ DUe to Biases in $\left(\ln H^{i}, \ln D_{A}^{i}\right)$.

\begin{tabular}{cccccc}
\hline \hline survey area & bias in $\ln D_{A}(z)$ & bias in $\ln H(z)$ & $\sigma_{z} /(1+z)$ & bias in $w_{0}\left(\sigma_{w_{0}}\right)$ & bias in $w^{\prime}\left(\sigma_{w^{\prime}}\right)$ \\
\hline $1000(\mathrm{deg})^{2}$ & $-1 \sigma$ & 0. & 0.001 & $0.082(0.115)$ & $-0.375(0.183)$ \\
& & & 0.01 & $0.255(0.266)$ & $-0.930(0.727)$ \\
& 0. & $1 \sigma$ & 0.02 & $0.273(0.396)$ & $-1.021(1.169)$ \\
\hline & & & 0.001 & $-0.181(0.115)$ & $0.317(0.183)$ \\
& & & 0.01 & $-0.303(0.266)$ & $0.491(0.727)$ \\
& & 0. & 0.02 & $-0.421(0.396)$ & $0.742(1.169)$ \\
\hline $10000(\mathrm{deg})^{2}$ & $-1 \sigma$ & & 0.01 & $0.033(0.069)$ & $-0.291(0.104)$ \\
& & $1 \sigma$ & 0.02 & $0.085(0.133)$ & $-0.311(0.251)$ \\
& 0. & & 0.001 & $-0.078(0.167)$ & $-0.317(0.386)$ \\
\hline & & & 0.01 & $-0.137(0.133)$ & $0.263(0.104)$ \\
& & & 0.02 & $-0.157(0.167)$ & $0.198(0.251)$ \\
& & & &
\end{tabular}




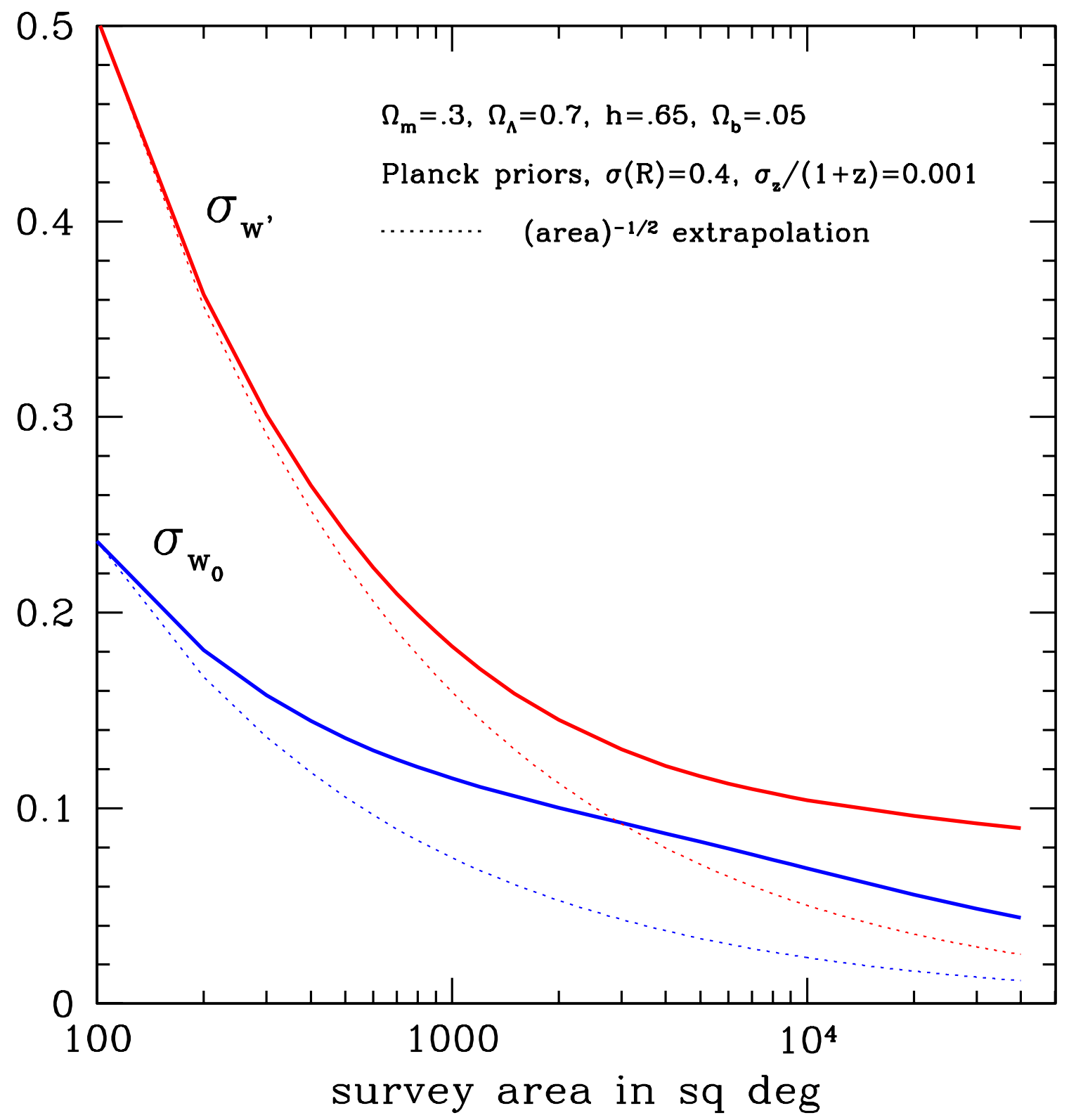

FIG. 1. - The $1 \sigma$ errors in $w_{0}$ and $w^{\prime}$ as function of survey area $(0.5 \leq z \leq 2)$. Note that the constraints on dark energy parameters scale much less steeply with survey area (for a given redshift range) than (area) ${ }^{-1 / 2}$ (which holds in the absence of the priors). 


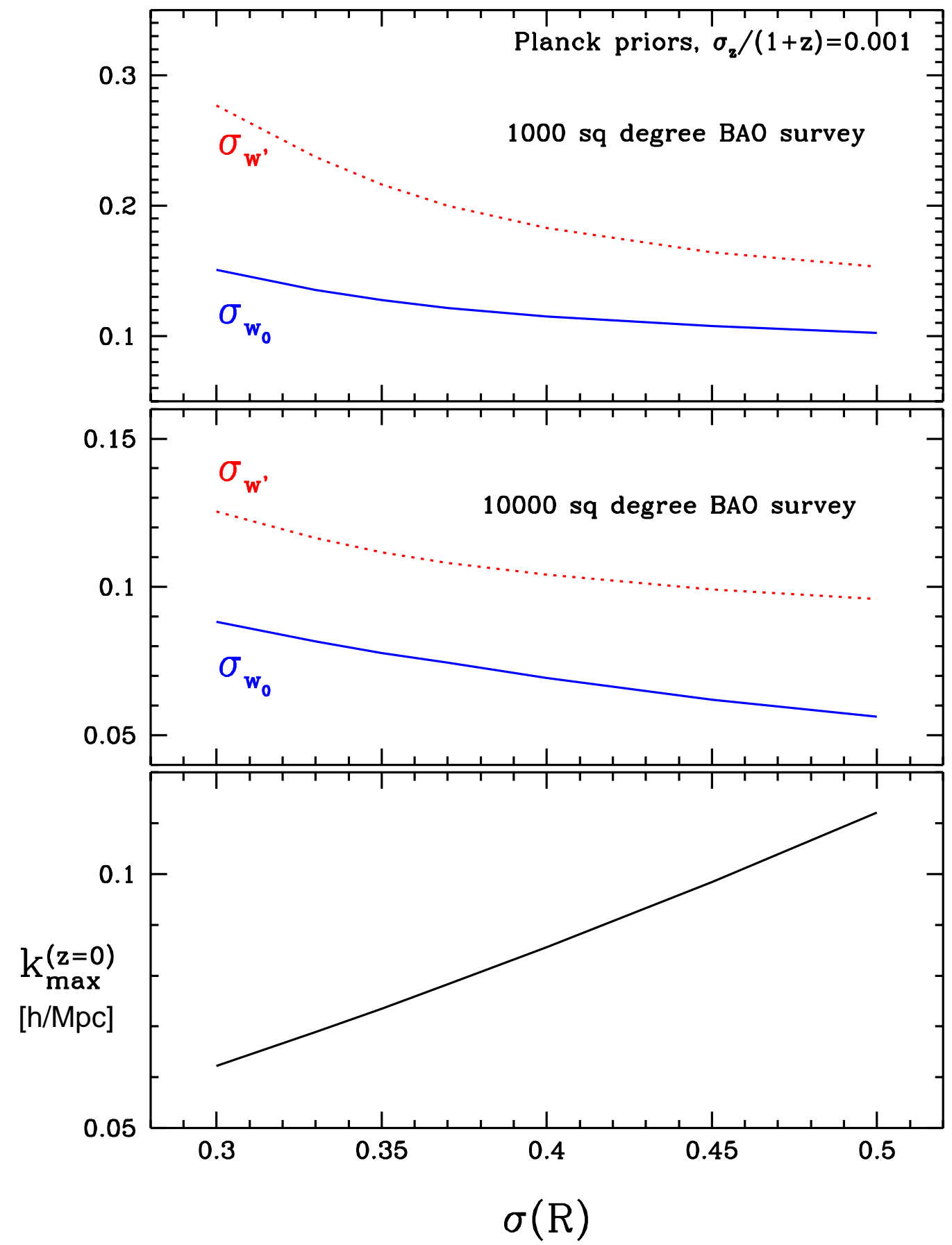

FIG. 2.- The $1 \sigma$ errors in $w_{0}$ and $w^{\prime}$ as function of $\sigma(R)$ for survey areas of 1000 and 10000 square degrees. The lowest panel gives the correspondence between $\sigma(R)$ and the cutoff wavenumber $k_{\max }$ at $z=0$. 


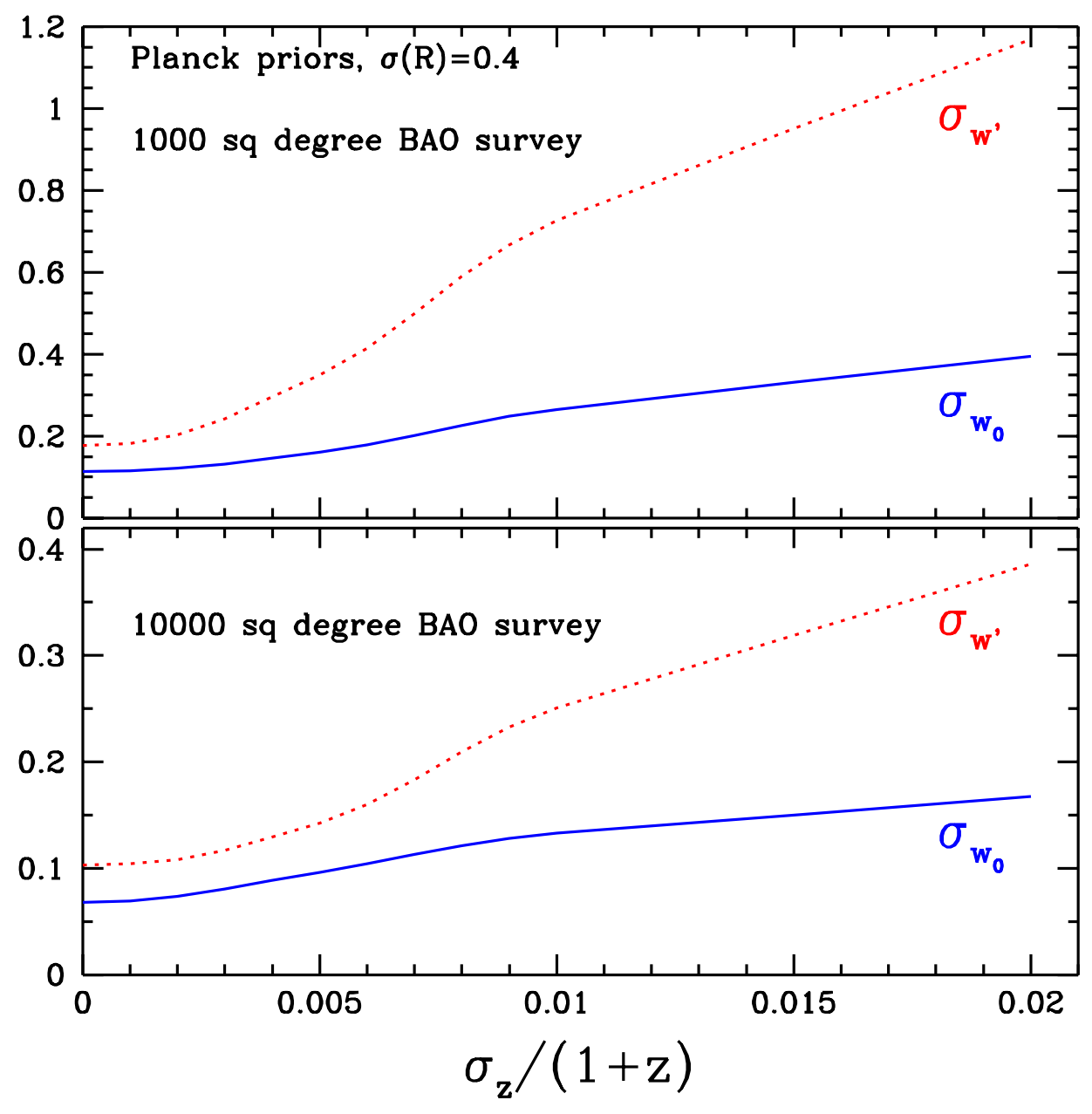

FIG. 3.- The $1 \sigma$ errors in $w_{0}$ and $w^{\prime}$ as function of $\sigma_{z} /(1+z)$ for survey areas of 1000 and 10000 square degrees. 


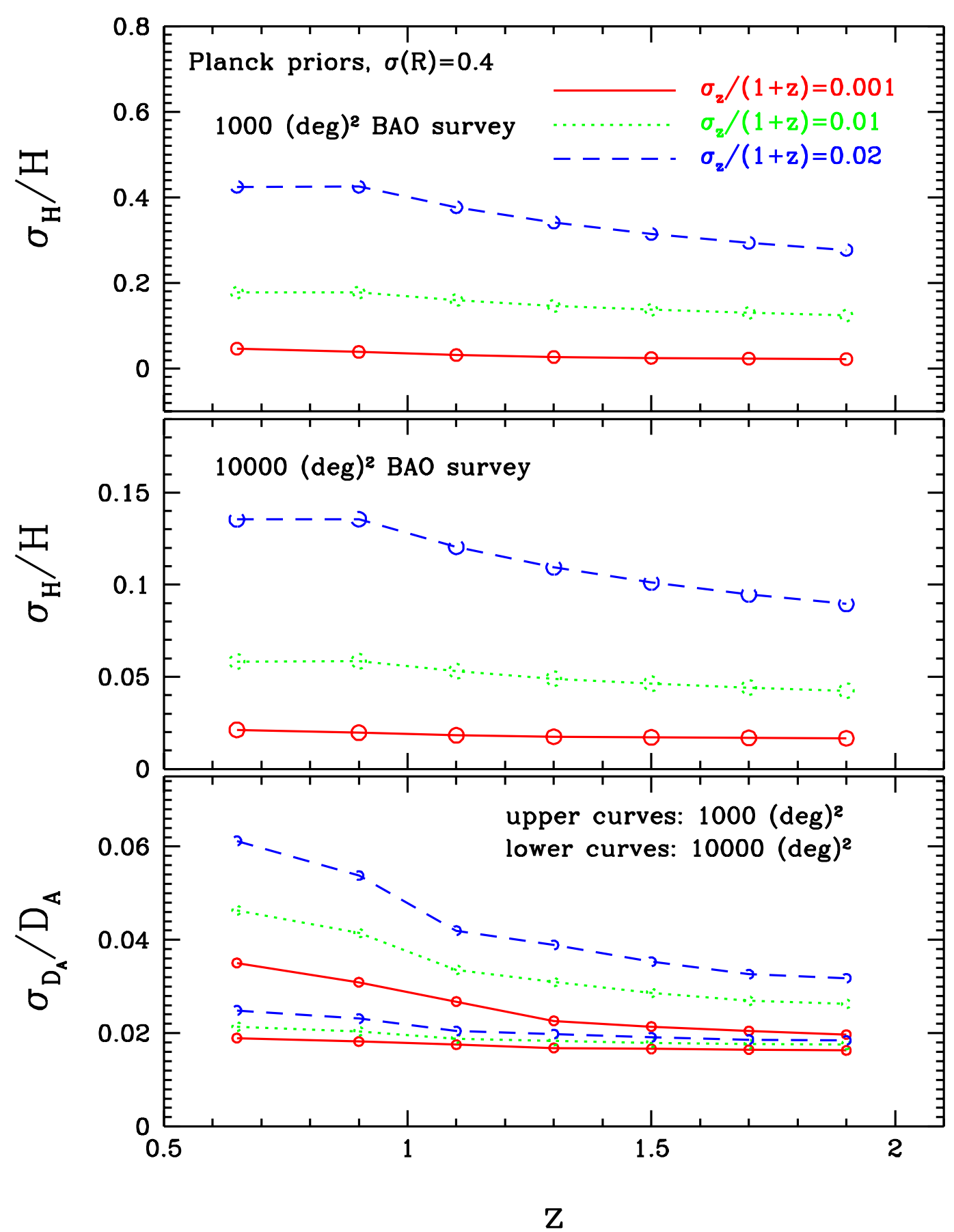

FIG. 4.- The estimated $\sigma_{H} / H$ and $\sigma_{D_{A}} / D_{A}$ in each of the redshift slices, for $\sigma_{z} /(1+z)=0.001,0.01$, and 0.02 for survey areas of 1000 and 10000 square degrees. 


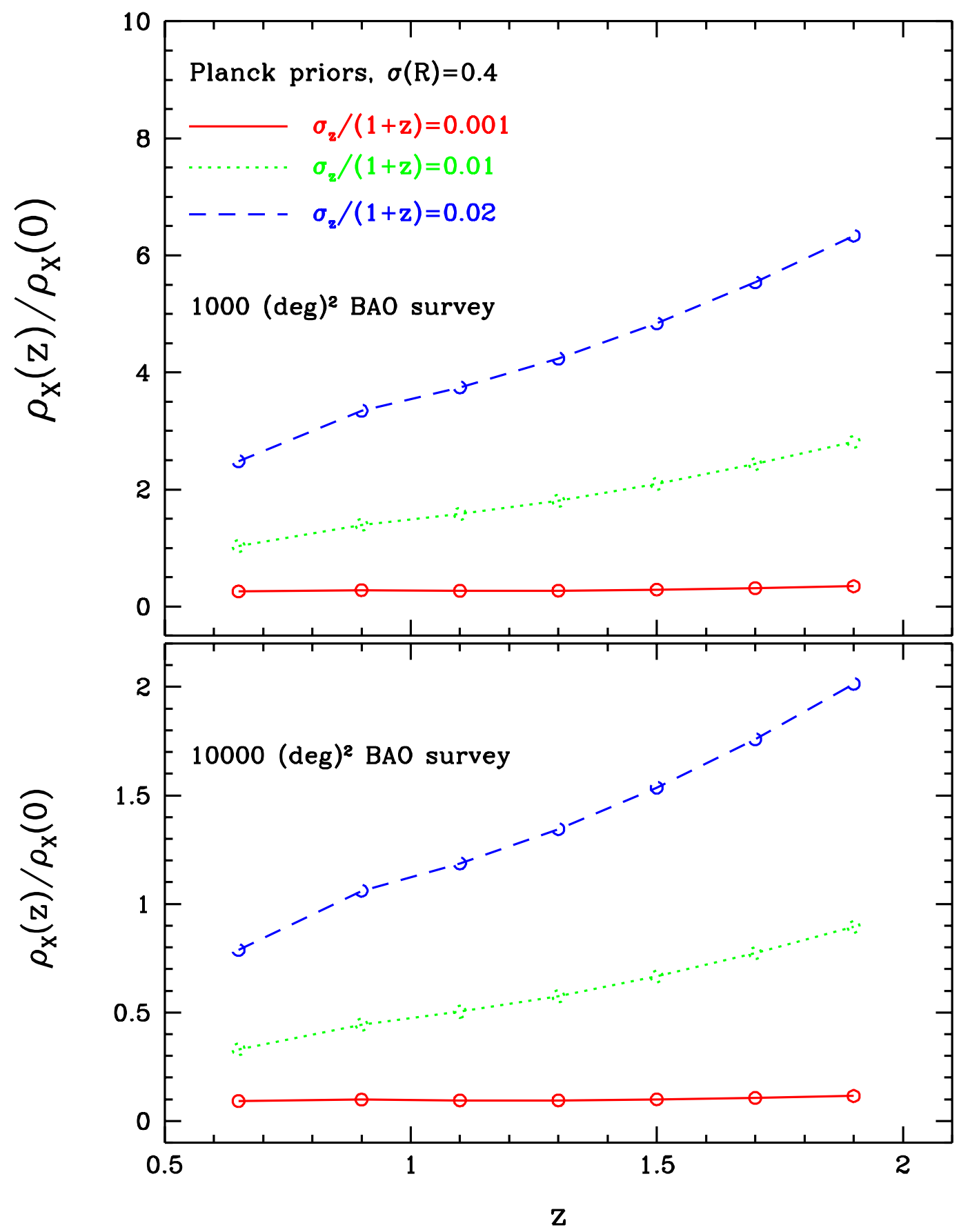

FIG. 5.- The estimated dark energy density $X(z)=\rho_{X}(z) / \rho_{X}(0)$ corresponding to Fig.4 (with the same assumptions and the same line types). 